\title{
Mid-and Long-term Survival and Patient-reported Outcomes of Humeral Surface Replacement in 135 Shoulders
}

Haider Mussawy ( $\boldsymbol{\sim}$ h.mussawy@uke.de)

Universitätsklinikum Hamburg-Eppendorf https://orcid.org/0000-0002-9511-163X

André Strahl

Universitatsklinikum Hamburg-Eppendorf

Tom Wenk

Klinikum Bad Bramstedt GmbH

Tobias Schmidt

Universitatsklinikum Hamburg-Eppendorf

Maciej Simon

Universitatsklinikum Hamburg-Eppendorf

Wolfgang Rüther

Universitatsklinikum Hamburg-Eppendorf

Research article

Keywords: Durom-Cup, humeral surface replacement, patient reported outcome, shoulder arthroplasty

Posted Date: August 6th, 2020

DOl: https://doi.org/10.21203/rs.3.rs-48844/v1

License: (9) (i) This work is licensed under a Creative Commons Attribution 4.0 International License.

Read Full License 


\section{Abstract}

Background. The aim of this retrospective study was to evaluate patient-reported clinical outcomes, prosthesis survival, and causes of revision of cemented humeral surface replacement (Durom-Cup) in a large population.

Methods. Between 1997 and 2015, 266 patients underwent 279 implantations of Durom-Cup. Overall, 81 patients died, 39 patients were lost to follow-up, and 21 patients were excluded according to pre-defined exclusion criteria, and dementia; this left 125 patients (135 shoulders) with a mean follow-up of 8.0 years (range, 1-20 years). To report long-term follow-up a subgroup of 51 patients with a minimum follow-up of 9 years and a mean follow-up of 12.4 years (range, 9-20 years) was evaluated. The mean age at the time of surgery was 65.8 years (range, 25-91 years).

Results. The indications were primary osteoarthritis (52\%) and rheumatoid arthritis (48\%). Patient satisfaction at final follow-up was as follows: very satisfied, 59.2\%; satisfied, $29.2 \%$; and not satisfied, $11.6 \%$. On a visual analogue scale (VAS) from 0 to 10 , the mean pain on weight-bearing was 2.4 (range, 0-10). Overall, $55.4 \%$ were able to perform leisure activities without major limitations. Patients with rheumatoid arthritis were significantly younger than patients with primary osteoarthritis at the time of surgery (mean difference, 11.5 years, $p<0.001$ ). The overall survival rates (prosthesis without revision surgery) of the Durom-Cup were $94.0 \pm 5.0 \%$ at 5 years, $90.8 \pm 9.86 \%$ at 10 years, and $90.8 \pm 17.1 \%$ at 15 years. However, 9 shoulders $(6.7 \%)$ required revision arthroplasty after a mean 4.3 years (range, $0.5-9$ years).

Conclusions. These data demonstrated a relatively low revision rate of the Durom-Cup and high satisfaction levels, suggesting an acceptable quality of life post-surgery.

\section{Background}

The main technical goal of shoulder arthroplasty is to reproduce the patient's normal anatomy. The main clinical goals of shoulder arthroplasty are pain relief and ability to perform activities of daily living (ADL).

Of the different endoprostheses available, cementless or cemented surface replacement of the humeral head, first described by Zippel [1] in 1975, has becoming less important; however, the operation time is shorter than for total shoulder arthroplasty or stemmed hemiarthroplasty [2], and the result is facile restoration of the anatomy and minimal bone resection, with low risk of periprosthetic fracture. Therefore, resurfacing allows preservation of bone stock should the need for revision arise in the future [3]. The main concern is that the revision rate is higher than that for total shoulder arthroplasty due to glenoid loss $[4,5]$.

Rydholm and Sjögren reported a loosening rate in $25 \%$, and proximal migration of the cup in $38 \%$, of 72 rheumatoid shoulders after 4.2 years; these rates had no relation on mobility, pain relief, or functional ability [6]. Similar results with the scan cup (strict hemispheric cup) were reported by Alund et al. [7] after 
a mean of 4.4 years; no difference was observed for the constant score between loosening and fixed prosthesis.

Two studies, published in 2004 [8] and 2007 [9], presented early results at 2 and 5 years postimplantation of a Durom-Cup in patients with rheumatoid arthritis (RA). The results of clinical evaluation in term of active range of motion and radiographic analysis were encouraging. The aim of the present study was to examine patient-reported outcomes in a large population after implantation of the DuromCup to either confirm or contradict previous study results with respect to prosthesis survival and revision rates.

\section{Methods}

\section{Study design and participants}

This study was conducted as a retrospective cohort study including patients who received a Durom-Cup humeral surface replacement (Fa. Zimmer, Winterthur, Switzerland) without glenoid component. The surgical technique for implanting a Durom-Cup was described in detail by Fuerst et al. [2]. Data were obtained from our hospital database. We extracted records for all patients over 18 years of age who underwent this surgical procedure between 1997 and 2015. Patients were included if they had primary osteoarthrosis $(\mathrm{OA})$ or secondary osteoarthrosis caused by RA. Further diseases were excluded due to the small number of cases (posttraumatic, $n=6$; cuff tear arthropathy, $n=5$; osteonecrosis of the humeral head, $n=3$; other medical indications, $n=3$ ) and poor comparability. The actual data collection was designed as a written questionnaire survey.

From the hospital database and according to the inclusion criteria, 249 patients were eligible to be included in the study. The latest contact details were provided by the responsible registration offices to increase the response rate and to obtain information about possible deaths. Finally, 168 patient-based questionnaires were sent out (81 patients had already passed away). The dropout rate was $25.6 \%$ (43 patients): 30 did not respond to the survey, nine had relocated and did not receive the questionnaire, and four were diagnosed with dementia and therefore were unable to complete the questionnaire. A total of 125 patients ( $\mathrm{n}=135$ shoulders) with humeral surface replacement participated in the survey (Fig. 1).

\section{Outcome measures}

The patient-based questionnaire covers five survey areas: revision surgery and service life of the DuromCup prosthesis; pain; impairment with respect to work and ADL; shoulder function; and satisfaction with surgery. Patient satisfaction with the surgical procedure and the overall result was measured on a 3-point ordinal rating scale. Information on revision was based on data provided by the patients in the questionnaire and on subsequent analysis of the patient's health records, as the revision surgery performed at the same institution. Pain was measured on a nominal scale comprising three categories: pain at night, pain at rest, and pain during active movement. When pain was indicated, it was quantified 
from 0 to 10 on a visual analogue scale (VAS). To measure shoulder impairment, participants were asked to score restrictions (categories = occupation and leisure) subjectively on a 5-point ordinal rating scale from "fully functional" to "no work or activity possible".

Shoulder function was measured in terms of five active clinical parameters: anteversion, abduction, external rotation, clasping the hand behind the waist, and operating height. Different gradations of these parameters were made with the aid of illustrative examples. Using this method, patients were able to indicate on the questionnaire the degree of movement they could achieve.

\section{Statistical analysis}

Statistical analysis was performed using the statistics package SPSS (version 25.0). The values of descriptive statistics are expressed as the mean expanded by minimum and maximum (range). Nominal data are expressed as number (percentage). To evaluate the distribution of gender within the sample a $\mathrm{Chi}^{2}$-test was calculated. Furthermore, a t-test for independent variables was used to test the difference in age at the time of surgery regarding the initial diagnoses (OA vs. RA). Survival probabilities, i.e. time to revision arthroplasty, were estimated by the Kaplan-Meier method. Potential risk factors (diagnosis gender) were calculated by the univariate Log-Rank test. In accordance with accepted standards, statistical significance was set to a 2-tailed p-value of 0.05 .

\section{Results}

\section{Study population}

Of the 125 patients enrolled, $51.9 \%$ were diagnosed with primary $\mathrm{OA}$, and $48.1 \%$ with RA. The mean age at the time of surgery was 65.8 years (range, 25.0-91.0). Unexpectedly, 73.6\% $(n=92)$ were women and only $26.4 \%(n=33)$ were men $\left(\mathrm{Chi}^{2}=28.3, \mathrm{p}<0.001\right)$. Patients with RA were 11.5 years younger than patients with OA at the time of surgery (59.8 vs. 71.3 years of age, respectively; $T=7.185, p<0.001)$. All demographic data are presented in Table 1. 
Table 1

demographic characteristics

\begin{tabular}{|ll|}
\hline & value \\
\hline gender, female, N (\%) & $91(74.0)$ \\
\hline age at surgery in years, M (SD) & $65.8(10.9)$ \\
\hline age at survey in years, M (SD) & $73.7(10.3)$ \\
\hline operated shoulder, N (\%) & $12(9.8)$ \\
- both sides & $69(56.1)$ \\
- right side & $42(34.1)$ \\
- left side & $70(51.9)$ \\
\hline diagnoses, N (\%) & $65(48.1)$ \\
primary osteoarthritis & \\
\hline secondary osteoarthritis related to rheumatoid arthritis
\end{tabular}

\section{Survival rate and complications related to the Durom-Cup}

The mean follow-up period for all implanted Durom-Cups was 8.0 years (range, 1-20 years). KaplanMeier survival analyses are presented in Fig. 2. The overall survival rates (prosthesis without revision surgery) of the Durom-Cup were $94.0 \pm 5.0 \%$ at 5 years, $90.8 \pm 9.86 \%$ at 10 years, and $90.8 \pm 17.1 \%$ at 15 years. The survival rates of the Durom-Cup were for OA $96.8 \pm 3.6 \%$ at 5 years, $91.9 \pm 15.8 \%$ at 10 years, and $91.9 \pm 37.5 \%$ at 15 years. The survival rates for RA were $91.7 \pm 3.9 \%$ at 5 years, $89.5 \pm 10.2 \%$ at 10 years, and $89.5 \pm 18.7 \%$ at 15 years. Therefore, no significant difference between implant survival rates in patients with OA or RA ( $\log$ Rank $\mathrm{Chi}^{2}=0.608, \mathrm{p}=0.435$ ) or between survival rates in women and men (Log Rank $\left.\mathrm{Chi}^{2}=0.027, \mathrm{p}=0.87\right)$ was observed. Fourteen patients $(10.5 \%)$ underwent revision; five of these underwent only soft tissue surgery due to joint stiffness, which is not a typical reason for revision in the literature. These operations took place within the first 2 years after implantation of the Durom-Cup (one case at 4 months, two cases at 7 months, one case at 8 months, one case at 12 months, and one case at 24 months post-surgery) and were not correlated with RA.

Nine patients (6.7\%) underwent revision of the Durom-Cup after a mean 4.3 years (range, $0.5-9$ years). Four patients were revised to a reverse arthroplasty due to glenoid erosion at $3,4,6$, and 8 years postimplantation of the Durom-Cup, and five patients were revised to an anatomical prosthesis. Of these five patients, three underwent revision due to loosening of the implant at 4, 5, and 6 years post-surgery, two due to prosthesis infection at 6 and 16 months post-surgery. Overall, $6 / 9$ patients in whom the prosthesis was revised had RA.

\section{Patient-reported outcomes}


The mean VAS pain score on weight-bearing was 2.4 (range, 0-10); there were no significant differences between patients with $\mathrm{OA}$ and RA or between women and men. Of the patients that reported pain-related sleep disturbance (41.1\%), 32.3\% reported "sometimes" and $8.9 \%$ reported "frequently".

Overall, $27.4 \%$ could participate in ADLs without any physical limitations. However, $1.8 \%$ of patients could not work at all and $1.7 \%$ were unable to participate in their favorite leisure activity. Detailed descriptive statistics are presented in Table 2 . At final follow-up, $59.2 \%$ of patients were very satisfied with the outcome, $29.2 \%$ were satisfied, and $11.7 \%$ were not satisfied (Table 2 ). 
Table 2

patient reported outcomes

\begin{tabular}{|ll|}
\hline outcome variable & value \\
\hline pain, N (\%) & $57(46.3)$ \\
- yes & $66(53.7)$ \\
- no & \\
\hline nature of pain, N (\%) & $21(36.8)$ \\
- pain at night & $1(1.8)$ \\
- pain at rest & $35(61.4)$ \\
- pain during stress & \\
\hline pain intensity (VAS 0-10), M (SD) & $2.4(2.9)$ \\
\hline limitations during work, N (\%) & $2(1.9)$ \\
- no work activity possible & $14(12.6)$ \\
- severe limitations & $31(27.9)$ \\
- moderate limitations & $21(18.9)$ \\
- minor limitations & $43(38.7)$ \\
\hline - no limitations & \\
\hline limitations during leisure time, N (\%) & $2(1.6)$ \\
- no leisure activity possible & $15(12.4)$ \\
- severe limitations & $37(30.6)$ \\
- moderate limitations & $25(20.7)$ \\
- minor limitations & $42(34.7)$ \\
- no limitations & $40(32.2)$ \\
\hline sleep disturbances due to shoulder complaints, N (\%) \\
- often & \\
- from time to time & \\
- no sleep disturbances & \\
\hline
\end{tabular}




\begin{tabular}{|ll|}
\hline outcome variable & value \\
\hline Overall satisfaction with the Durom-Cup prosthesis, N (\%) & $71(59.2)$ \\
- very satisfied & $35(29.2)$ \\
- satisfied & $14(11.6)$ \\
\hline - not satisfied & \\
\hline
\end{tabular}

\section{Functional outcome parameters}

All functional parameters are presented in Fig. 3. Shoulder functionality was measured in terms of anteversion, abduction, external rotation, clasping the hand behind the waist, and operating height. Overall, $66.3 \%$ of the patients had an anteversion $>120^{\circ}$, although $14.5 \%$ reported $<60^{\circ}$, which we defined as "severe impairment". Abduction $>120^{\circ}$ was reported by $56.7 \%$ of patients, but $22.1 \%$ reported $<60^{\circ}$. External rotation was achieved without difficulty by $66.0 \%$ of patients (i.e., they could raise the hand above the head while keeping the elbow wide and away from the body). Clasping the hands behind the waist showed the poorest results; only $42.0 \%$ of the patients were able to clasp the hands behind the back and above the belt (most of them were able to place the hands on the belt or only to the lower edge of the back trouser pockets). $45.5 \%$ of patients could operate with the hand on the head; i.e. comb their hair.

\section{Survival rate and patient-reported outcomes in a subgroup of at least 9 years follow-up}

Additionally, all patients with a minimum follow-up of 9 years were evaluated separately. These 51 shoulders had a mean follow-up of 12.4 years (range, 9-20 years). Only one of these 51 (2.0\%) shoulders was revised because of symptomatic glenoid erosion. The prosthesis was switched to a reverse arthroplasty. Of these 51 shoulders $48.0 \%$ reported to have any pain with a mean intensity of 2.2 (VAS range, $0-8)$. When pain was reported it occurred on weight-bearing (50.0\%) and during night $(50.0 \%)$. Pain at rest was not reported. $34.0 \%$ had pain related sleep disturbance. At final follow-up, $63.3 \%$ of patients were very satisfied with the outcome and $59,6 \%$ had no or only few restrictions in the ADL.

\section{Discussion}

Here, we report patient-reported mid- and long-term outcomes after implantation of a Durom-Cup. Of the 135 studied shoulders that received a Durom-Cup, only 9 underwent revision of the implant over a mean follow-up period of 8.0 years. These data, together with few intraoperative complications, suggest that cemented surface replacement remains an alternative solution for shoulder replacement in elderly patients with $O A$, particularly in patients with RA.

Previous studies report conflicting results after surface replacement. We and others previously demonstrated good clinical outcomes for patients with RA $[9,10]$. Of interest, patients in the present cohort with different pathologies had similar clinical outcomes. Patients with RA were on average 
11.5 years younger than those with primary osteoarthritis, as might be expected. There was no difference between the groups in terms of pain on weight-bearing or shoulder functionality. There was no significant difference in functional outcome and implant survival rates between men and women. It is notable that 6/9 revision operations were in patients with secondary osteoarthritis caused by RA and may be due to glenoid erosion.

In terms of functional outcome, more than $50 \%$ of patients in the study group had few restrictions with respect to work (57.6\%) and leisure activities (55.4\%), whereas nearly $30 \%$ had moderate restrictions. In total, $88.3 \%$ of patients were satisfied with the outcome of the operation. However, this level of satisfaction corresponds with the outcome reported by patients that received the Copeland prosthesis. Rai et al. [11] reported a satisfaction rate of $88 \%$ at 12 years $(n=34$ shoulders receiving the Copeland mark III), whereas Bailie et al. [12] reported a rate of $97.2 \%(n=35$ patients aged $<55$ years receiving the Copeland mark II) at 2 year follow-up. The high satisfaction rate reported in the latter study is likely due to the short follow-up and young age at the time of operation.

The implant-associated revision rate reported herein was $6.7 \%$. This is comparable to that in the current literature. Although Rai et al. reported a revision rate of $4 \%$, Levy et al. reported a rate of $5-6 \%$ after $5-$ 10 years [13]. Alizadehkhaiyat et al. reported a revision rate $>20 \%$ at a mean follow-up of 4 years [14], whereas Soudy et al. [15] reported a revision rate (surface replacement to reverse shoulder arthroplasty) of $17.5 \%$ (18/105 patients) after a mean follow-up of 43.6 months; the total revision rate was $23 \%$ (24/105 patients). Data from the Danish Shoulder Register show a cumulative revision rate of $14 \%$ for resurfacing hemiarthroplasty and only $2 \%$ for stemmed hemiarthroplasty after a mean of 5 years [16]. Thus, the danish authors suggest that stemmed prosthesis designs may offer better implant survival than resurfacing. For example, Barlow et al. (2014) reported a revision rate of $7 \%$ after 10 years for total shoulder arthroplasty in RA patients, which is comparable to the revision rate for cemented surface replacement reported herein. The main reason for revision after total shoulder arthroplasty was glenoid loosening and infection [17]. Different results were reported by Maier et al. (2018); they described a very high revision rate after cementless surface replacement ( $24 \% ; n=8$ patients, 34 shoulders) at 2.7 years. In all eight cases, the reason for revisions was glenoid erosion.

The intraoperative complication rate associated with surface replacement is quite low when compared with that reported by other studies of shoulder arthroplasty. The largest study to date was published by Cowling et al. [18]. This study of a national data set comprising 12,559 primary shoulder arthroplasties reported an intraoperative complication rate of $2.5 \%$. Significantly fewer complications occurred in patients undergoing resurfacing arthroplasty than in those undergoing total shoulder arthroplasty, reverse shoulder arthroplasty, or stemmed hemiarthroplasty.

Due to the patient-reported outcome study we cannot draw definitive conclusions regarding complications due to proximal migration, glenoid erosion, or radiolucent lines under the cup; if the patients had no symptoms. The majority of our patients had low pain levels during the day and sufficient range of motion to undertake activities of daily living. 
This retrospective patient-reported outcome study does not permit us to state that patients experienced clinical improvement. Nevertheless, to the best of our knowledge, this study represents the largest population of patients with implanted cemented humeral resurfacing in the literature. Furthermore, patient-reported outcomes might be of more relevance [19] than radiological follow-up, particularly in elderly patients more concerned with pain relief, functionality, and improved ability to undertake work and leisure activities.

\section{Conclusion}

The present study reports mid-and long-term follow-up of cemented humeral surface replacement. The results demonstrated a relatively low revision rate and high satisfaction levels, suggesting an acceptable quality of life post-surgery.

\section{Abbreviations}

\section{Durom Cup}

cemented humeral surface replacement; ADL:activities of daily living; RA:rheumatoid arthritis; OA:primary osteoarthrosis; VAS:visual analogue scale;

\section{Declarations}

\section{Ethics approval and consent to participate}

The study was performed in accordance with the Declaration of Helsinki (1975), as revised in 2000. Ethical approval was obtained from the local ethics committee of the University Medical Center HamburgEppendorf (registration number: PV5037). All included patients provided written informed consent.

\section{Consent for publication}

Not applicable.

\section{Availability of data and materials}

The dataset used and analyzing during the current study are available from the corresponding author on reasonable request.

\section{Competing interests}

HM, AS, TW, TS, MS and WR declare that they have no conflict of interest concerning this article and report no financial support.

\section{Funding}

The study was not supported by any funding or grants. 


\section{Acknowledgements}

Not applicable.

\section{Authors' contributions:}

Study design: HM, TS, MS, WR. Study conduct: HM, AS, TW, WR. Data collection: TW, TS, MS. Data analysis: HM, AS. Drafting manuscript: HM. Approving final version of manuscript: HM, AS, TW, TS, MS, WR. All authors have read and approved the manuscript.

\section{References}

1. Zippel J. [Dislocation-proof shoulder prosthesis model BME]. Z Orthop Ihre Grenzgeb. 1975;113(4):454-7. PubMed PMID: 1210534.

2. Fuerst $M$, Fink $B$, Ruther $W$. The DUROM cup humeral surface replacement in patients with rheumatoid arthritis. Surgical technique. J Bone Joint Surg Am. 2008;90 Suppl 2 Pt 2:287-98. doi: 10.2106/JBJS.H.00312. PubMed PMID: 18829941.

3. Fink B, Strauss JM, Lamla U, Kurz T, Guderian H, Ruther W. [Endoprosthetic surface replacement of the head of the humerus]. Orthopade. 2001;30(6):379-85. PubMed PMID: 11480090.

4. Gartsman GM, Roddey TS, Hammerman SM. Shoulder arthroplasty with or without resurfacing of the glenoid in patients who have osteoarthritis. J Bone Joint Surg Am. 2000;82(1):26-34. PubMed PMID: 10653081.

5. Hasan SS, Leith JM, Campbell B, Kapil R, Smith KL, Matsen FA, 3rd. Characteristics of unsatisfactory shoulder arthroplasties. J Shoulder Elbow Surg. 2002;11(5):431-41. PubMed PMID: 12378161.

6. Rydholm U, Sjogren J. Surface replacement of the humeral head in the rheumatoid shoulder. J Shoulder Elbow Surg. 1993;2(6):286-95. doi: 10.1016/1058-2746(93)90074-Q. PubMed PMID: 22971788.

7. Alund M, Hoe-Hansen C, Tillander B, Heden BA, Norlin R. Outcome after cup hemiarthroplasty in the rheumatoid shoulder: a retrospective evaluation of 39 patients followed for 2-6 years. Acta Orthop Scand. 2000;71(2):180-4. doi: 10.1080/000164700317413166. PubMed PMID: 10852325.

8. Fink B, Singer J, Lamla U, Ruther W. Surface replacement of the humeral head in rheumatoid arthritis. Arch Orthop Trauma Surg. 2004;124(6):366-73. doi: 10.1007/s00402-004-0661-x. PubMed PMID: 15133697.

9. Fuerst $M$, Fink $B$, Ruther $W$. The DUROM cup humeral surface replacement in patients with rheumatoid arthritis. J Bone Joint Surg Am. 2007;89(8):1756-62. doi: 10.2106/JBJS.F.01290. PubMed PMID: 17671015.

10. Thomas SR, Wilson AJ, Chambler A, Harding I, Thomas M. Outcome of Copeland surface replacement shoulder arthroplasty. J Shoulder Elbow Surg. 2005;14(5):485-91. doi: 10.1016/j.jse.2005.02.011. PubMed PMID: 16194739. 
11. Rai P, Davies O, Wand J, Bigsby E. Long-term follow-up of the Copeland mark III shoulder resurfacing hemi-arthroplasty. J Orthop. 2016;13(1):52-6. doi: 10.1016/j.jor.2015.09.003. PubMed PMID: 26955225; PubMed Central PMCID: PMCPMC4761623.

12. Bailie DS, Llinas PJ, Ellenbecker TS. Cementless humeral resurfacing arthroplasty in active patients less than fifty-five years of age. J Bone Joint Surg Am. 2008;90(1):110-7. doi: 10.2106/JBJS.F.01552. PubMed PMID: 18171964.

13. Levy O, Copeland SA. Cementless surface replacement arthroplasty of the shoulder. 5- to 10-year results with the Copeland mark-2 prosthesis. J Bone Joint Surg Br. 2001;83(2):213-21. PubMed PMID: 11284568.

14. Alizadehkhaiyat O, Kyriakos A, Singer MS, Frostick SP. Outcome of Copeland shoulder resurfacing arthroplasty with a 4-year mean follow-up. J Shoulder Elbow Surg. 2013;22(10):1352-8. doi: 10.1016/j.jse.2013.01.027. PubMed PMID: 23562291.

15. Soudy K, Szymanski C, Lalanne C, Bourgault C, Thiounn A, Cotten A, et al. Results and limitations of humeral head resurfacing: 105 cases at a mean follow-up of 5 years. Orthop Traumatol Surg Res. 2017;103(3):415-20. doi: 10.1016/j.otsr.2016.12.015. PubMed PMID: 28167247.

16. Voorde PC, Rasmussen JV, Olsen BS, Brorson S. Resurfacing shoulder arthroplasty for the treatment of severe rheumatoid arthritis: outcome in 167 patients from the Danish Shoulder Registry. Acta Orthop. 2015;86(3):293-7. doi: 10.3109/17453674.2015.1018761. PubMed PMID: 25673155; PubMed Central PMCID: PMCPMC4443462.

17. Barlow JD, Yuan BJ, Schleck CD, Harmsen WS, Cofield RH, Sperling JW. Shoulder arthroplasty for rheumatoid arthritis: 303 consecutive cases with minimum 5-year follow-up. J Shoulder Elbow Surg. 2014;23(6):791-9. doi: 10.1016/j.jse.2013.09.016. PubMed PMID: 24315477.

18. Cowling PD, Holland P, Kottam L, Baker P, Rangan A. Risk factors associated with intraoperative complications in primary shoulder arthroplasty. Acta Orthop. 2017;88(6):587-91. doi: 10.1080/17453674.2017.1362155. PubMed PMID: 28782448; PubMed Central PMCID: PMCPMC5694801.

19. Deshpande PR, Rajan S, Sudeepthi BL, Abdul Nazir CP. Patient-reported outcomes: A new era in clinical research. Perspect Clin Res. 2011;2(4):137-44. doi: 10.4103/2229-3485.86879. PubMed PMID: 22145124; PubMed Central PMCID: PMCPMC3227331.

\section{Figures}


eligibly shoulders with DUROM Cup Humeral Surface Replacement $\mathrm{n}=262$ (249 patients)

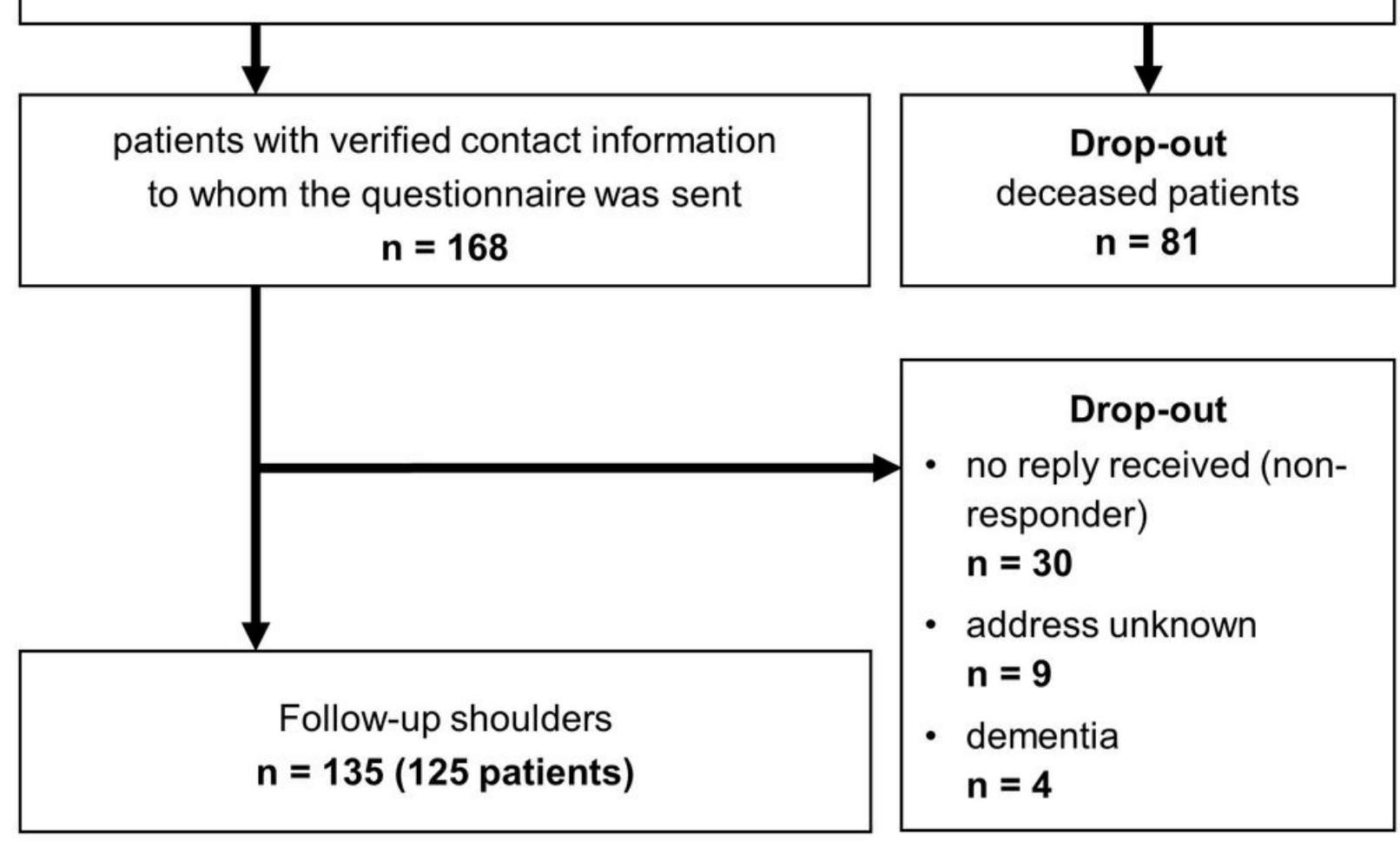

Figure 1

Study profile. Number of patients taking part in the study, along with details of dropouts. 


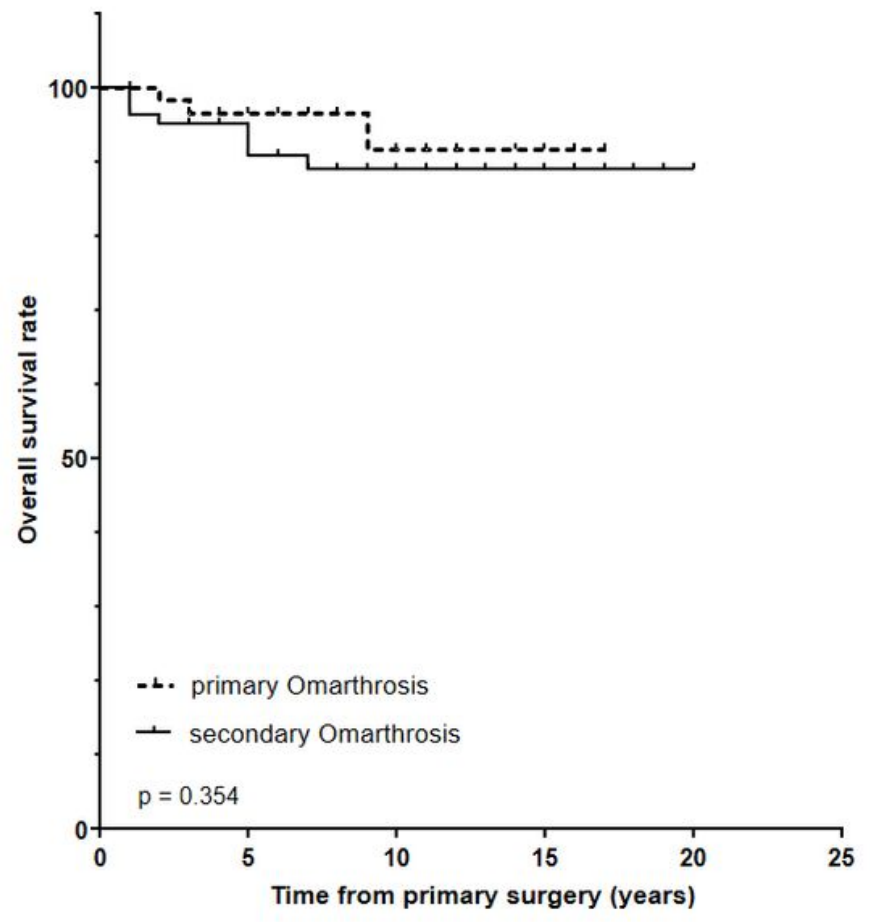

\section{Figure 2}

Overall survival of the Durom-Cup shoulder prosthesis (years). Prosthesis survival is compared between patients with OA and RA (log-rank test, $\mathrm{p}<0.05$ ). 
A

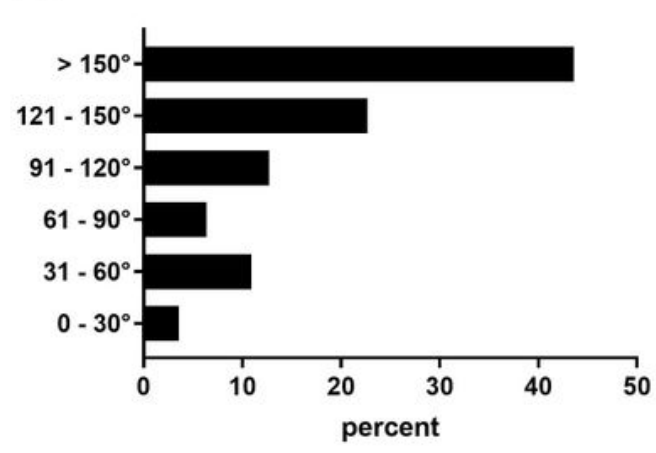

C

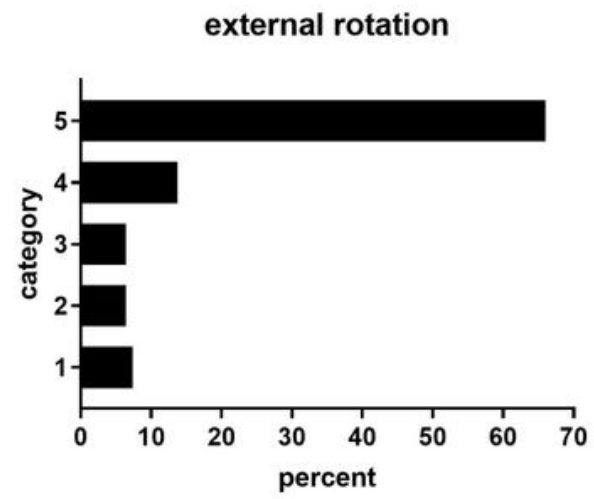

${ }^{1}$ hand clasping the neck / elbow facing forward

${ }^{2}$ hand on top of the head / elbow facing forward

${ }^{3}$ hand clasping the neck / elbow to the side

${ }^{4}$ hand on the head / elbow to the side

${ }^{5}$ hand over the head / elbow to the side

E

clasping the hands behind the waist

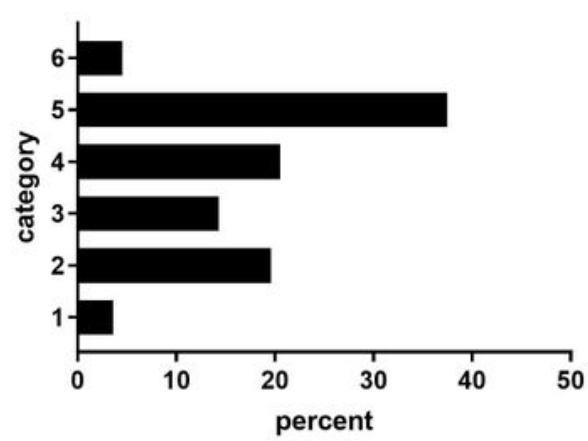

1 not possible

${ }^{2}$ up to the beginning of the back trouser pockets

${ }^{3}$ up to under the belt

${ }^{4}$ up to the belt

${ }^{5}$ up to over the belt

${ }^{6}$ up between the shoulder blades
B abduction

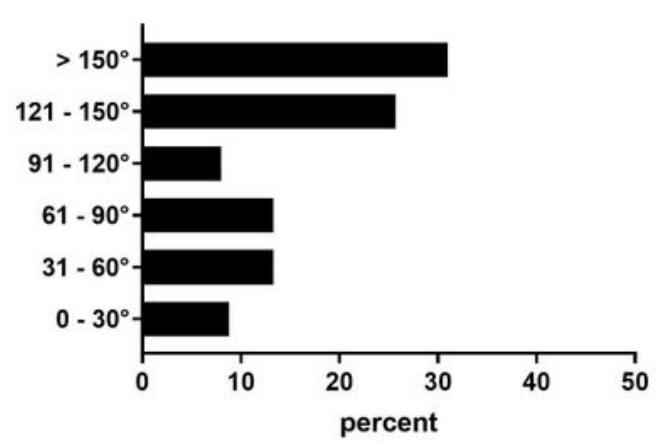

D

operating height

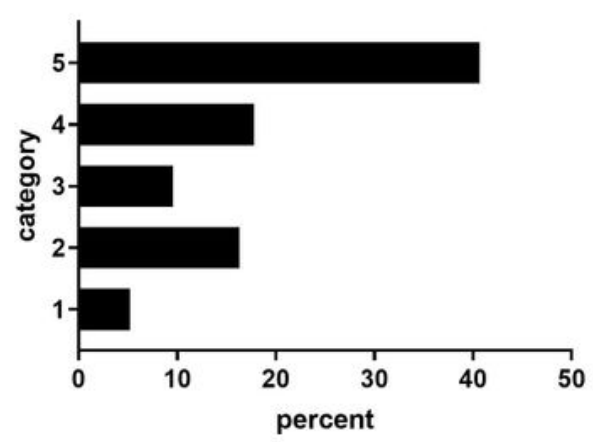

1 up to the waist

${ }^{2}$ up to the chest

${ }^{3}$ up to the neck

${ }^{4}$ up to the head

${ }^{5}$ upside the head

\section{Figure 3}

Patient-reported functional parameters. Patients were asked to perform specific movements using standardized images presented in the questionnaire. (A) Anteversion in six different "degrees of motion" categories. (B) Abduction in six different "degrees of motion" categories. (C) External shoulder rotation (five categories). (D) Possible operating height (five categories). (E) Clasping hands behind the waist (five categories). 